\title{
Group-based parent training programmes demonstrate positive effects on the psychosocial well-being of both mothers and fathers
}

\section{WHAT IS ALREADY KNOWN ON THIS TOPIC?}

A previous meta-analysis by the same first author focused on parent training using data on mothers found that parent training programmes improved mothers' psychosocial well-being, including depression, anxiety/stress, self-esteem and spouse/marital adjustment. ${ }^{1}$

\section{WHAT THIS PAPER ADDS?}

- Previous findings for mothers were replicated. Postintervention, parent training programmes significantly decreased maternal depression, anxiety, stress, anger, guilt, and improved maternal confidence and satisfaction with the partner relationship. Yet only maternal stress and confidence continued to be statistically significant at short-term follow-up (1-6 months), and none were significant at long-term follow-up (more than 6 months).

- Parent training programmes significantly reduced paternal stress postintervention.

\section{LIMITATIONS}

- Publication bias needs to be addressed, because small studies with non-significant result effects usually do not get published.

- Outcomes at three different time points were presented; yet different studies were included at each point of analysis. Furthermore, very few studies had long-term follow-up data. For example, regarding maternal depressive symptoms, data analysis included 22 studies for postintervention, 13 for short-term and 7 for long-term follow-up. While included in the analysis for the shortterm and long-term analysis, Hiscock et al's ${ }^{2}$ study with the largest sample size and negative outcomes was not included with the postintervention analysis. This might have impacted the results.
- Authors did not report Cls in the main text when the results were not significant. Study results should be interpreted in terms of the width of $\mathrm{Cl}$, not statistical significance.

\section{WHAT NEXT IN RESEARCH}

Parent training programmes reduced child psychosocial problems through the promotion of effective parenting. ${ }^{3}$ Future studies should include the associations between parent and child outcomes using correlations between two outcomes controlling for baseline. Sorting studies based on effect size and exploring the features of intervention may help to find types of intervention that work better than others. Which intervention would work better when parents have psychosocial issues: psychosocial intervention or parent training? Parent training programmes that include management of parenting stress may have different effect from those that do not include this content. The effects of culturally tailored parent training should also be explored.

\section{DO THESE RESULTS CHANGE YOUR PRACTICES AND WHY?}

Yes, the results can be used to change clinical practice to include psychosocial assessment of both parents, to educate both parents in positive parenting practices and to refer to parent training, when indicated. At a systems level, more community-based parent training programmes should be developed.

Competing interests None.

doi:10.1136/eb-2014-101929

- For reference list, see online version of article at http://ebmh.bmj.com

ABSTRACT FROM: Barlow J, Smailagic N, Huband N, et al. Group-based parent training programmes for improving parental psychosocial health. Cochrane Database Syst Rev 2014;5:CD002020.

Data sources CENTRAL, MEDLINE, EMBASE, CINAHL, BIOSIS, PsycINFO, Sociological Abstracts, Social Science Citation Index, ASSIA, ERIC, NSPCC and metaRegister of Controlled Trials up to 5 December 2011. Reference lists were hand searched for relevant studies.

Study type included Randomised controlled trials (RCTs) and quasi-RCTs. Intervention Group-based parent training programmes that help parents to manage children's behaviour and improve family functioning, relationships and psychosocial health. Training programmes were based on cognitive behavioural, behavioural, multimodal or other approaches.

Control Waiting list, no treatment, treatment as usual or a placebo.

\section{OUTCOMES}

Study characteristics Forty-eight RCTs ( $\mathrm{n}=4937)$ were identified, 22 conducted in the USA; 10 in Australia; 7 in Canada; 3 in the UK; and the reminder in China, Germany, Japan, the Netherlands and New Zealand. Outcomes were by self-report using a variety of standardised tools. Comparisons between intervention and control groups were made immediately after the intervention, in the short-term ( 6 months) and long-term (1 year or more) follow-ups.

Depressive symptoms Assessed by 29 studies, using six different scales. In pooled analysis of 22 studies, parent training programmes improved depressive symptoms compared with control immediately after the intervention (standard mean difference $(\mathrm{SMD})=-0.17,95 \% \mathrm{CI}-0.28$ to -0.07 ). There was no statistically significant difference at short-term or long-term follow-up.

Anxiety symptoms Assessed by 13 studies, using five different scales. In pooled analysis of nine studies, parent training programmes improved anxiety symptoms compared with control immediately after the intervention $(\mathrm{SMD}=-0.22,95 \% \mathrm{CI}-0.43$ to -0.01$)$. There was no significant difference at short-term or long-term follow-up.

Stress Assessed by 36 studies, using nine different scales. In pooled analysis of 25 studies, parent training programmes improved stress compared with control immediately after the intervention $(\mathrm{SMD}=-0.29,95 \% \mathrm{CI}-0.42$ to $-0.15)$. They also improved stress at short-term follow-up ( $\mathrm{SMD}=-0.22$, $95 \%$ CI -0.42 to-0.01), but no difference was seen at long-term follow-up. Anger Pooled analysis of three studies found that parent training programmes improved anger compared with control immediately after the intervention ( $\mathrm{SMD}=-0.60,95 \% \mathrm{CI}-1.00$ to -0.20 ). No studies reported short-term or long-term effects.

Guilt Pooled analysis of three studies found that parent training programmes improved feelings of guilt compared with control immediately after the intervention ( $\mathrm{SMD}=-0.79,95 \% \mathrm{CI}-1.18$ to -0.41$)$. No studies reported short-term or long-term effects. 